\title{
New 2015 American College of Rheumatology (ACR) Rheumatoid Arthritis Guidelines
}

\author{
Salah Eldin Abdel Baky \\ Department of Internal Medicine, Division of Rheumatology, Ain Shams University; Egypt
}

\begin{abstract}
During a very well attended session at the ACR meeting, a panel of speakers discussed the $2015 \mathrm{draft}$ guidelines for rheumatoid arthritis (RA) treatment that are expected to be published in early 2015 .

There were a recommendations provided in 2008 and then in 2012, so I would have expected another 4 years before any additional information was provided. This is earlier than anticipated. Perhaps this suggests an acceleration in the progress of our understanding of rheumatoid arthritis and its management

The updated guidelines review newer agents, including DMARDS, biologics, the oral Janus kinase inhibitor tofacitinib and, "for the first time," glucocorticoids. The updated recommendations also considered concomitant conditions associated with RA, including prior cancer, infection, tuberculosis, hepatitis, and congestive heart failure, as well as the safety of vaccinations and when to discontinue and/or taper therapy.
\end{abstract}

"The panel strongly recommended a treat-totarget strategy, in both early and established rheumatoid arthritis. For many patients the target will be low disease activity based on patient comorbidity and risk tolerance.

In early rheumatoid arthritis - defined as a disease duration of less than 6 months - the panel strongly recommended disease-modifying antirheumatic drug (DMARD) monotherapy for DMARD-naive patients with low, moderate, or high disease activity.

For patients with moderate or high disease activity who have a flare, the panel recommended glucocorticoid use at the lowest possible dose for the shortest period.

Patients who fail DMARD monotherapy and still have moderate to high disease activity should receive a combination of traditional DMARDs, a tumor necrosis factor (TNF) inhibitor with or without methotrexate, or a non-TNF-inhibitor biologic with or without methotrexate.

If these patients experience a rheumatoid arthritis flare, short-term glucocorticoids are again recommended.

The panel recommended adding low-dose glucocorticoids to the treatment regimen if these combination options are not effective and disease activity remains moderate to high.

For Correspondence: email salahym50@yahoo.com
In established rheumatoid arthritis, the treatment algorithm for is similar to that recommended for early disease.

For DMARD-naive patients with established disease, DMARD monotherapy, usually methotrexate is recommended as first-line treatment for those with low, moderate, or high disease activity.

After DMARD failure, the panel strongly recommends the combination of traditional DMARDs, TNF inhibitor with or without methotrexate, nonTNF-inhibitor biologic with or without methotrexate, or tofacitinib plus methotrexate.

If disease activity remains moderate to high in these patients, physicians can consider adding lowdose glucocorticoids. Short-term glucocorticoids can also be used for rheumatoid arthritis flares.

Patients who fail a single TNF inhibitor should next receive a non-TNF-inhibitor biologic with or without methotrexate, or another TNF inhibitor with or without methotrexate. Those who fail on a nonTNF inhibitor biologic should receive another nonTNF-inhibitor biologic with or without methotrexate.

\section{Introducing Tofacitinib}

Next in line for those who fail multiple TNF inhibitors is a non-TNF-inhibitor biologic with or without methotrexate or tofacitinib with or without methotrexate.

Patients who fail a TNF inhibitor and non-TNFinhibitor biologic should receive another non-TNFinhibitor biologic with or without methotrexate or tofacitinib with or without methotrexate.

Those who fail treatment with several non-TNFinhibitor biologics should receive tofacitinib with or without methotrexate or a TNF inhibitor with or without methotrexate, provided they have not previously received a TNF inhibitor.

\section{High-Risk Patients, Complicated Cases}

Solid Tumor Malignancies: In 2012, use of biologics in the setting of solid malignancy was categorized as greater than 5 years after treatment with biologic agents, but was confined to rituximab with malignancy less than 5 years, treated melanoma or treated lymphoproliferative malignancies. In 2014, the 5-year limitation has been lifted, and the following changes were made: 
- Non-melanoma skin cancer \& RA treated with DMARDs or non-TNF biologics over TNFi

- Melanoma skin cancer \& RA treated with TNFi over tofacitinib

- $\quad$ Previously treated lymphoproliferative disorders with DMARDS or a non-TNF biologic over TNFi

- Patients with previously treated solid organ malignancy are now recommended to be treated the same as patients without this condition

Hepatitis C: In 2012, treatment of hepatitis C was limited to the use of etanercept in 2012. However, in 2014, DMARDs, TNF inhibitors (TNFi), non-TNF biologics or tofacitinib are now listed as recommended.

Hepatitis B: Untreated or treated hepatitis B had no recommended treatment in 2012; biologic agents were included as "not recommended." By contrast, in 2014, patients with active hepatitis B infections and receiving effective anti-viral treatment could be treated with DMARDs, TNFi, non-TNF biologic or tofacitinib as recommended interventions.

Congestive heart failure (CHF): In the 2012 guideline, there are no recommendations for the management of NYHA Class III/IV CHF in RA patients, but anti-TNF agents are deemed to be "not recommended." In 2014, combination DMARDS or non-biologic therapy or tofacitinib are recommended over TNFi, but TNFi are no longer listed as "not recommended."

Serious infection: In the 2012 guideline, the issue of serious infection is not addressed. However, in 2014, combination DMARD is recommended over
TNFi, and abatacept over TNFi but TNFi are not listed as "not recommended." Further, there is a lack of consensus on the role of either rituximab or tocilzumab over TNFi. Interestingly enough, no comment is directed towards tofacitinib.

Patients 50 years and older should receive the herpes zoster vaccine before biologic therapy. However, live attenuated vaccines, such as the herpes zoster vaccine, should not be given to patients with early or established rheumatoid arthritis if they are on any biologic therapy.

The panel also strongly recommended continuing therapy with traditional DMARDs, TNF inhibitor, non-TNF-inhibitor biologics, or tofacitinib in patients with established rheumatoid arthritis and low disease activity who are continuing on methotrexate.

For those in remission and continuing on methotrexate, the panel recommended that physicians taper treatment, regardless of the regimen.

"At the same time, the panel strongly recommended that not all therapies be discontinued, because "clinical experience suggests that only a very small minority of patients with established rheumatoid arthritis are able to discontinue all treatment."

\section{REFERENGES}

1. American College of Rheumatology (ACR) 2014 Annual Meeting. Presented November 16, 2014.Boston.

2. http://www.rheumatology.org/session select

3. www.medscape.com. Pam Harrison. November 27,2014 